\title{
Assembly of platforms for signal transduction in the new era: dimerization, helical filament assembly, and beyond
}

\author{
Hyun Ji Ha', Hye Lin Chun ${ }^{1}$ and Hyun Ho Park $\mathbb{D}^{1}$
}

\begin{abstract}
Supramolecular organizing center (SMOC)-mediated signal transduction is an emerging concept in the field of signal transduction that is ushering in a new era. The formation of location-specific, higher-order SMOCs is particularly important for cell death and innate immune signaling processes. Several protein interaction domains, including the death domain (DD) superfamily and the CIDE domain, are representative mediators of SMOC assembly in cell death and innate immune signaling pathways. DD superfamily- and CIDE domain-containing proteins form SMOCs that activate various caspases and provide signaling scaffold platforms. These assemblies can lead to signal transduction and amplification during signaling events. In this review, we summarize recent findings on the molecular basis of DD superfamily- and CIDE domain-mediated SMOC formation.
\end{abstract}

\section{Introduction}

Specific protein interactions are critical for proper cellular signaling, and failed and mismatched protein interactions cause signaling defects and alter the fate of cells. Protein interaction is usually mediated by protein interaction domains, a critical part of proteins involved in the binding of specific sequences to other proteins ${ }^{1,2}$.

Cell death and innate immune signaling pathways are important defense mechanisms against various pathogens. These processes are mediated by various complicated protein-protein interactions that transfer signals and control cellular signaling events. Many proteins participating in these cellular signaling events contain small protein interaction domains, such as the death domain (DD), death effector domain (DED), caspase-recruiting domain (CARD), PYrin domain (PYD), baculovirus IAP repeat (BIR) domain, Bcl-2 homology (BH) domain, and/ or cell death-inducing DFF45-like effector (CIDE) domain. These proteins interact with specific binding partners $^{3-5}$. Among the protein interaction domains, DD,

\footnotetext{
Correspondence: Hyun Ho Park (xrayleox@cau.ac.kr)

${ }^{1}$ College of Pharmacy, Chung-Ang University, Seoul 06974, Republic of Korea These authors contributed equally: Hyun Ji Ha, Hye Lin Chun
}

DED, CARD, and PYD belong to the death domain (DD) superfamily. This is one of the largest protein interaction domain families, sharing sequence homology and a unifying structural feature: a six-helix bundle fold ${ }^{3,6-8}$.

Apoptotic DNA fragmentation is a hallmark of apoptosis and is primarily mediated by the CIDE domaincontaining proteins DFF40 and DFF $45^{9}$. DFF40 is an endonuclease that digests chromosomal DNA and produces nucleosomal fragments, whereas DFF45 is an inhibitor of DFF40 ${ }^{10}$. Both DFF40 and DFF45 contain a CIDE domain that can mediate the interaction between two proteins, resulting in the inhibition of DFF40 nuclease activity by DFF $45^{11,12}$. In addition to DFF40/DFF45, the CIDE-A, CIDE-B, and CIDE-3 proteins have been identified as having CIDE domains ${ }^{11,13}$. Although functional studies have shown that these three CIDE domaincontaining proteins are also involved in apoptosis regulation, recent studies have indicated their role in energy metabolism, specifically their involvement in controlling the size of lipid droplets ${ }^{14-16}$.

Over recent decades, the interprotein interactions in the cell death and innate immune signaling pathways mediated by DD superfamily-containing and CIDE 
domain-containing proteins have been intensively studied. This interest is based on their functional importance in biological systems and their links to many human diseases, including cancer, obesity, and various immune diseases ${ }^{7,17-22}$. Studies have revealed that various signaling molecules in cell death and innate immune signaling form higher-order signaling complexes called supramolecular organizing centers (SMOCs) via DD superfamily or CIDE domains ${ }^{23,24}$. In addition to SMOC formation, DD superfamily-containing proteins can be assembled into various oligomerization structures. In this review, we summarize the binding strategies of the DD superfamily and CIDE domains detected thus far. We also discuss the biological significance of these assemblies during cell death and innate immune signaling events.

\section{Structure and function of the DD superfamily}

In the early 1990s, an intracellular DD comprising 90 amino acids was first identified and named during a cellular study on tumor necrosis factor receptor and $\mathrm{Fas}^{25-27}$. Since then, genetic, functional, and structural analyses have revealed similar DD-like domains in various proteins, designated DEDs ${ }^{28}, \mathrm{CARDs}^{29}$, and PYDs ${ }^{30,31}$. The subfamily classification is mainly determined by sequence homology ${ }^{6}$. In humans, 37 DD-containing proteins, 7 DED-containing proteins, 33 CARD-containing proteins, and 22 PYD-containing proteins have been identified thus far and found to be particularly functional during cell death and innate immunity events ${ }^{4,6}$. DD superfamily-containing proteins specifically interact through their DDs with other downstream DD superfamily-containing proteins, thus transferring signals through cellular signaling. In addition, DD superfamily-mediated SMOC formation is critical for activating various caspases and kinases, which are necessary for cell death and innate immunity processes ${ }^{32,33}$.

The six-helix bundle fold is the common feature of the DD superfamily (Fig. 1a). The structure of the Fas DD, with the six-helix bundle fold, was the first structure among the DD superfamily-containing proteins to be identified $^{25}$. Then, the structures of the Fas-associated DD protein (FADD) with a DED $^{28}$; RIP-associated protein with DD (RAIDD) with a CARD ${ }^{34}$; and NACHT, leucinerich repeat and PYD-containing 1 (NLRP1) with a PYD ${ }^{35}$ were elucidated (Fig. 1a). Although they possess a common structural fold, each subfamily has unique structural features, including a flexible and exposed third helix (H3) in DDs, an RxDL motif in DEDs, a bent first helix (H1) in CARDs, and a relatively small $\mathrm{H} 3$ and long $\mathrm{H} 2-\mathrm{H} 3$ connecting loop in PYDs (Fig. 1a).

\section{Prototype interactions of the DD superfamily}

Each member of the DD superfamily interacts with members of its own subfamily ${ }^{3,8,36-38}$. The general strategy of DD superfamily assembly was revealed via a structural study of the PIDDosome core complex, a molecular complex that activates caspase- 2 and is composed of RAIDD DD and PIDD DD ${ }^{7}$. This structural study showed that seven RAIDD DD and five PIDD DD molecules formed a circular, three-layer structure: two layers are formed by RAIDD DD and one is formed by PIDD DD (Fig. 1b). The main two layers, the bottom layer and middle layer, are formed by five PIDD DDs and five RAIDD DDs, respectively, whereas the top layer is formed by two additional RAIDD DDs (Fig. 1b). This circular structure is formed by the unique rotation and translation strategy of DD molecules. A planar schematic showed that the circular complex is constructed by five successive screw rotations of the DD molecules in the same layer around the central vertical axis. One screw rotation rotates approximately $84^{\circ}$ and translates down the axis, and the other rotates approximately $54^{\circ}$ and translates up the axis. These segments can form a circular structure with three $84^{\circ}$ and two $54^{\circ}$ rotations $((84 \times 3)+(54 \times 2)=$ $360^{\circ}$ ) (Fig. 1b). This unique circular structure formed by DDs participates in three types of interactions, namely, types I, II, and III, which are now considered the prototype interactions of the DD superfamily (Fig. 1b). In type I interactions, residues in the $\mathrm{H} 1$ and $\mathrm{H} 4$ helices of one DD interact with residues in the $\mathrm{H} 2$ and $\mathrm{H} 3$ helices of the bound DD (Fig. 1c). In type II interactions, an interface is formed by residues in the $\mathrm{H} 4$ helix and the $\mathrm{H} 4-\mathrm{H} 5$ loop of one DD and the residues in the H5- H6 loop and H6 helix of the bound DD (Fig. 1c). In type III interactions, residues in the $\mathrm{H} 3$ in one $\mathrm{DD}$ and the residues in the connecting loops, from $\mathrm{H} 1-\mathrm{H} 2$ and $\mathrm{H} 3-\mathrm{H} 4$, of the bound DD. This interaction strategy formed by the hetero DD complex was also detected in members of other subfamilies of the DD superfamily. The representative heterodimeric CARD structure of the Apaf-1 CARD and caspase-9 CARD complex revealed that the interaction is mediated by the mutual recognition of the concave surface formed by $\mathrm{H} 1$ and $\mathrm{H} 4$ in the caspase- 9 CARD and the convex surface formed by $\mathrm{H} 2$ and $\mathrm{H} 3$ in the Apaf- 1 CARD. These interactions indicate that this heterodimeric CARD structure is constructed via typical type I interactions (Fig. 1d) ${ }^{39}$. Type I-mediated homodimer CARD assembly was also discovered via a structural study of ARC $\mathrm{CARD}^{40}$. The homodimer interface of ARC CARD is created by salt bridges and hydrogen bonds formed between the residues in $\mathrm{H} 1$ and $\mathrm{H} 4$ of one ARC CARD and the residues in $\mathrm{H} 2$ and $\mathrm{H} 3$ of its counterpart. The type IImediated dimeric DD structure was discovered by a structural study of Pelle DD and Tube DD (Fig. 1e) ${ }^{41}$. In this case, $\mathrm{H} 4$ and the H4-H5 loop in Tube DD interact with the H5-H6 loop in Pelle DD. Further structural study of the complex formed by DDs revealed that a similar assembly strategy, based on prototype type I, type II, and type III interactions, occurs during Fas DD/FADD DD 


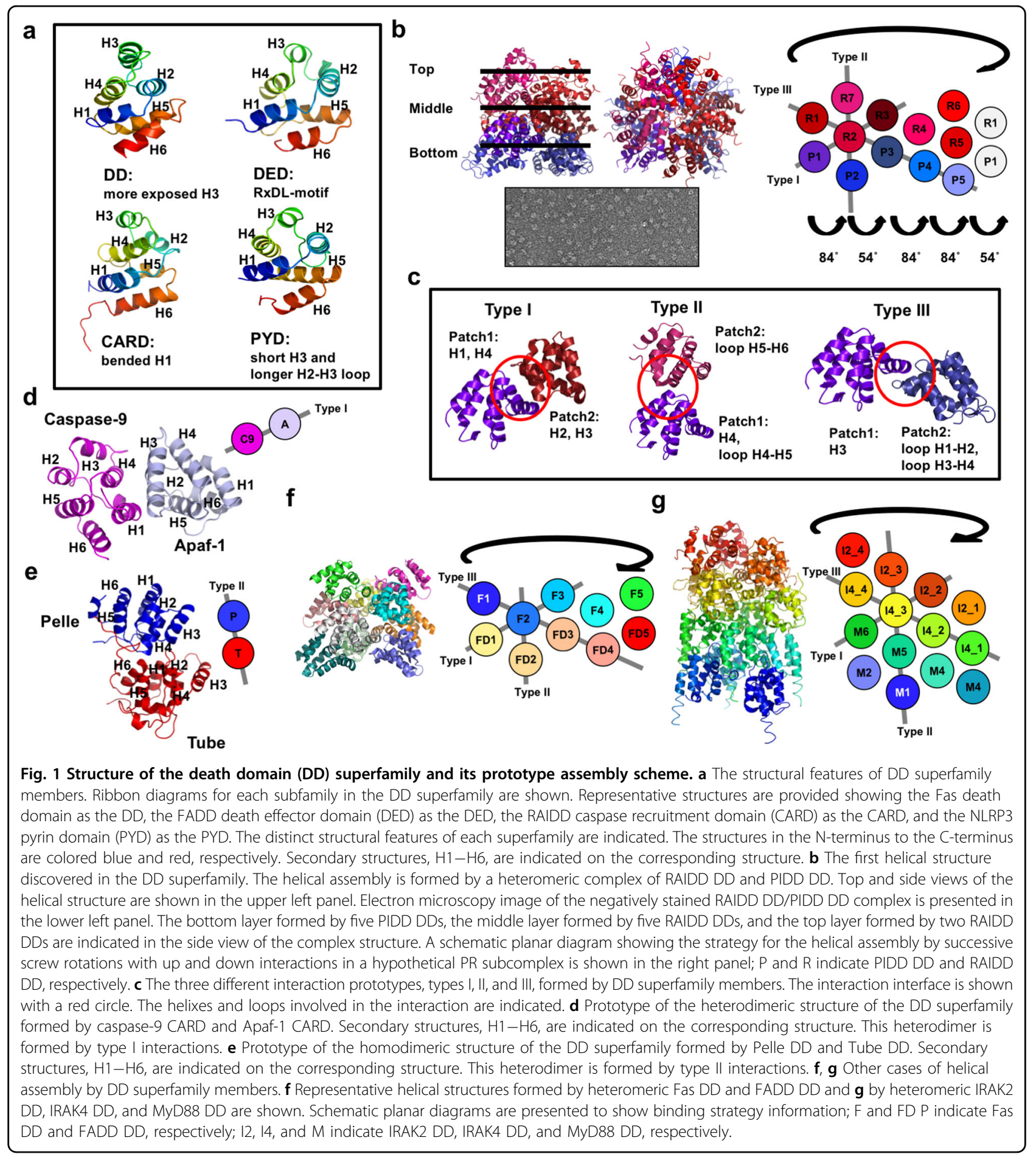

complex formation (Fig. 1f) ${ }^{17,42}$, RIP1DD/FADD DD complex formation ${ }^{42}$, and MyD88 DD/IRAK4 DD/IRAK2 DD complex formation (Fig. $1 \mathrm{~g})^{20}$. This suggests that the type I-, type II-, and type III-mediated assembly strategies are common among DD superfamily members. However, atypical dimeric structures in members of the DD superfamily were also found in recent structural studies ${ }^{43-45}$.

\section{SMOC formation by the DD superfamily}

The large molecular complexes formed by the helical assembly of DD superfamily members were difficult to determine by their crystal structure because it is impossible to crystallize a helical structure unless the helical periodicity happens to be an integer. With the development of advanced cryo-electron microscopy (EM) 
technology, many helical filament structures of the DD superfamily, important for SMOC formation, have been determined $^{18,19,21,46-50}$. These structural studies revealed that members of the DD superfamily use a common assembly mechanism, detected during the structural study of the RAIDD DD/PIDD DD complex, to form the helical filament structure in SMOCs. This mechanism is formed via type I, II, and III interactions.

DD superfamily-mediated SMOC formation has been studied most intensively via structural studies of an inflammasome composed of Nod-like receptor (NLR), inflammatory caspase-1, and the ASC adaptor molecule $^{51-55}$. Each NLRP family protein and caspase-1 contains a PYD and CARD at their respective N-terminus. ASC is a bipartite adaptor containing a PYD and CARD, which can link NLRP with caspase-1 by mediating the interaction of PYD-PYD through NLRP and the interaction of CARD-CARD through caspase-1. The results of an analysis based on cryo-EM indicated that all members of the DD superfamily in the inflammasome form a helical filament structure $\mathrm{e}^{19,21,48,56}$.

The ASC CARD forms a helical filament with a diameter of $\sim 8 \mathrm{~nm}$ and a central hole of $\sim 1 \mathrm{~nm}$ (Fig. $2 \mathrm{a})^{19}$. The filament architecture is assembled via left-handed one-start helical symmetry with approximately 3.6 subunits per turn. It is formed by three types of typical asymmetric interactions and displays threefold symmetry along the helical axis (Fig. 2a). Another CARD filament is introduced by caspase-1 to form a helical filament with a left-handed one-start helical assembly with approximately four subunits per turn. This structure is similar to the filament structure of ASC CARD (Fig. 2b) ${ }^{21}$. A caspase-1 CARD filament is also constructed using the three previously defined types of asymmetric interactions and has a diameter of $8 \mathrm{~nm}$ with an inner hole of approximately $1 \mathrm{~nm}$, which is slightly larger than that of the ASC CARD $^{21}$. ASC PYD also forms a helical filament using three types of canonical interaction modes. The filament is a right-handed helical filament with threefold symmetry along the helical axis (Fig. 2c) ${ }^{48}$. Although the diameter of the ASC PYD filament is similar to that of ASC CARD and caspase-1 CARD, the size of its inner hole was much greater (Fig. 2c). Based on the filament structures of the ASC CARD, caspase-1 CARD, ASC PYD, and NLRP PYD, the overall structure of the inflammasome and the assembly mechanism are proposed ${ }^{48,57,58}$. NLRP activation by a pathogen-associated molecular pattern triggers NLRP PYD assembly and filament formation, thus mediating ASC filament formation by serving as a nucleation platform via the PYD-PYD interaction. The helical filament of ASC recruits procaspase-1 via the CARD-CARD interaction. The recruited procaspase- 1 also forms a helical filament in the inflammasome complex, which mediates proximity-induced self-activation ${ }^{48,57,58}$.
The only helical filament structure of DED was revealed via a structural study of the tandem DED in caspase-8, which is activated in the death-inducing signaling complex (DISC) ${ }^{18}$. The overall structure and construction strategies of the helical DED filament, formed by typical type I, II, and III interactions, are similar to those of other subfamilies. However, the DED filament diameter of $\sim 9 \mathrm{~nm}$ is slightly greater than that of other filaments, and the right-handed helix does not have a distinct symmetry (Fig. 2d). In addition to these representative helical filament structures of the DD superfamily, various cases have been reported to date and are summarized in Table 1.

\section{Domain-swapping-mediated dimerization of DD superfamily members}

Domain swapping is widely used by proteins for the functional interconversion of monomers, dimers, and higher oligomers. It is also utilized by DD superfamily members for dimerization ${ }^{59-64}$. Domain-swappingmediated dimerization has been observed in all subfamilies of the DD superfamily, including Fas $\mathrm{DD}^{22}$, NLRP14 PYD ${ }^{65}$, caspase-8 DED ${ }^{66,67}$, and vPOP CARD ${ }^{68}$. Based on structural studies, it was revealed that two different domain-swapping mechanisms can be used to form a stable DD superfamily dimer. The first mechanism involves stem helix (formed by connected $\mathrm{H} 5$ and H6)mediated domain swapping, during which structural changes occur in the $\mathrm{H} 5$ and $\mathrm{H} 6$ regions. The stem helix is formed by $\mathrm{H} 5$ connecting to $\mathrm{H} 6$, which can interact with the stem helix through its binding counterpart. The DD and PYD subfamily members also use this mechanism for domain swapping. The details of this stem helix-mediated oligomerization were described in a structural study on the core of the DISC, which is composed of Fas DD/ FADD DD (Fig. 3a) ${ }^{22}$. In this study, a structural alteration was observed in the $\mathrm{H} 5$ and $\mathrm{H} 6$ regions of the Fas DD, which formed a stem helix and mediated domain swapping. This was the critical step for Fas DD dimerization and for the binding of Fas DD to FADD DD to form the DISC core. This stem helix-mediated domain swapping that leads to Fas DD dimerization was suggested to be an important regulatory mechanism for DISC formation. Stem helix formation that leads to domain swapping was also found during a structural study of NLRP14 PYD ${ }^{65}$. Similar to the structural alteration of the Fas DD, NLRP14 PYD forms a combined H5-H6 stem helix, which mediates the dimerization of NLRP14 PYD (Fig. 3b). Although the interface of the stem helix during the dimerization of NLRP14 PYD is not related to that of the Fas DD, the structural change-mediated dimerization strategy is similar to that utilized by Fas DD in the DISC. This similarity is based on the stem helix formed by $\mathrm{H} 5$ and H6, which is critical for generating the dimer interface. NOD1 CARD is also dimerized using this mechanism ${ }^{69}$. 
a

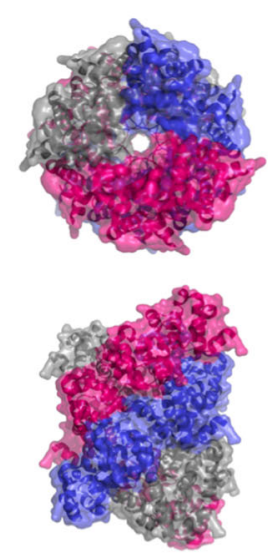

C

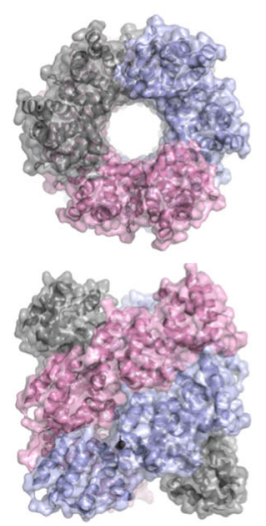

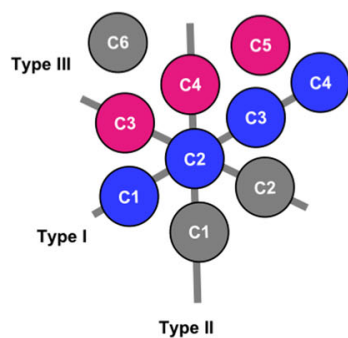

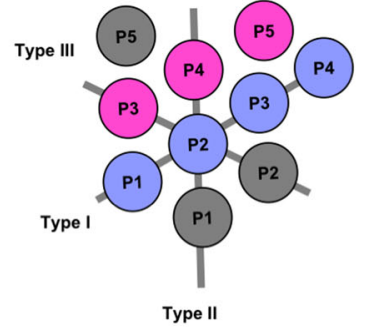

b

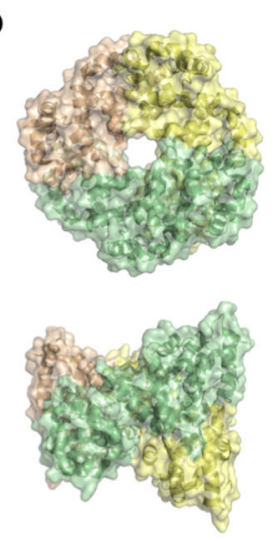

d

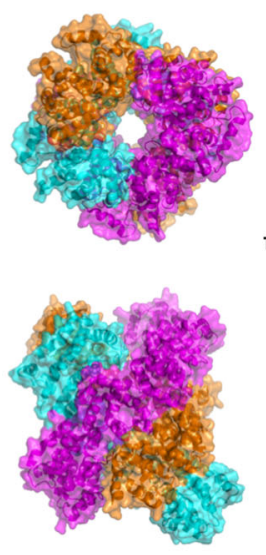

[Caspase-1 CARD]

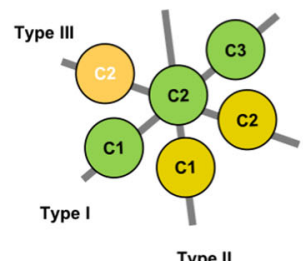

[Caspase-8 DED]

Fig. 2 Helical filament structure of the death domain (DD) superfamily. Helical filament structures of a the ASC caspase recruitment domain (CARD), $\mathbf{b}$ caspase-1 CARD, $\mathbf{c}$ the ASC pyrin domain (PYD), and $\mathbf{d}$ the caspase-8 death effector domain (DED) are represented. The surface structures are shown in the left panel from a topside view. A schematic planar diagram is shown in the right panel. The three different types of interactions used for helical filament formation are designated type I, type II, and type III. C and P in the schematic model indicate CARD (C) and PYD (P), respectively. The tandem DEDs of caspase-8 that produce a helical filament structure are separately indicated by D1 for the first DED and D2 for the second DED in the schematic model.

Another domain-swapping mechanism used by the DD superfamily is regional structural alteration-mediated local domain swapping, as demonstrated via structural studies of the DED and CARD subfamilies. In these cases, large structural changes involving the unfolding and extension of the $\mathrm{H} 3-\mathrm{H} 6$ region that allow for domain swapping were observed ${ }^{70,71}$. This dimerization strategy was shown in a structural study of viral CARD-only protein $(\mathrm{vCOP})^{68}$. In this structure, $\mathrm{H} 4, \mathrm{H} 5$, and $\mathrm{H} 6$ in the typical six-helix vCOP bundle are displaced and inserted between $\mathrm{H} 1, \mathrm{H} 2$, and $\mathrm{H} 3$ in a molecule opposite of vCOP. This insertion forms the typical six-helix bundle structure, leading to vCOP dimerization (Fig. 3c). Another case of huge structural change-mediated domain swapping and dimerization in the DD superfamily was discovered during a structural study of tandem DEDs in caspase- $8^{66,67}$. This study showed that the helical bundle of DED2 is unfolded from $\mathrm{H} 4$ to $\mathrm{H} 6$ and translocated to a counterpart molecule where it interacts with the $\mathrm{H} 1, \mathrm{H} 2$, and $\mathrm{H} 3$ helix bundles in the original $\mathrm{H} 4-\mathrm{H} 6$-contributing molecule, forming a new six-helix bundle (Fig. 3d). Both DED1 and DED2 in the caspase-8 tandem DEDs have an FL motif; it has been established that the FL motif in DED2 is critical for dimerization, oligomerization, and the further activation of caspase-8, whereas the FL motif in DED1 is not associated with either oligomerization or caspase activation $^{72-74}$. This result explains the reason that the FL motif in DED2 is critical for caspase-8 activation, showing that it is the primary mediator for caspase- 8 domain swapping and dimerization (Fig. 3e). By observing the domainswapping-mediated dimerization of caspase-8, a model of DISC assembly and caspase- 8 activation was suggested ${ }^{66}$. DISC assembly via the death ligand interaction with the death receptor, followed by FADD recruitment, mediates the accessibility of the FL motif in procaspase-8 DED2. This induced accessibility is followed by domain swapping, dimerization, and the activation of caspase-8. Because the trimeric death ligand and death receptor 
Table 1 Structures of various forms of DD superfamily.

\begin{tabular}{|c|c|c|c|c|c|}
\hline $\begin{array}{l}\text { DD } \\
\text { superfamily }\end{array}$ & Proteins & Monomer & Dimer & Filament & $\begin{array}{l}\text { Domain } \\
\text { swapping }\end{array}$ \\
\hline \multirow[t]{21}{*}{ DD } & FADD & $\mathrm{O}(2 \mathrm{GF5})$ & & $(30 Q 9)^{a}$ & \\
\hline & Fas & $\mathrm{O}$ (1DDF) & & $(30 Q 9)^{\mathrm{a}}$ & $\mathrm{O}(3 \mathrm{EZQ})$ \\
\hline & RAIDD & $\mathrm{O}(2 \mathrm{O} 71)$ & & $(2 \mathrm{OF} 5)^{\mathrm{a}}$ & \\
\hline & PIDD & & & $(2 \mathrm{OF} 5)^{\mathrm{a}}$ & \\
\hline & TRADD & O (5XME) & & & \\
\hline & RIP1 & $O(6 A C 5)$ & & & \\
\hline & IRAK-2 & & & $(3 \mathrm{MOP})^{\mathrm{a}}$ & \\
\hline & IRAK-3 & O (5UKE) & & & \\
\hline & IRAK-4 & $\mathrm{O}(2 \mathrm{~A} 9 \mathrm{I})$ & & $(3 \mathrm{MOP})^{\mathrm{a}}$ & \\
\hline & Ankyrin-1 & $\mathrm{O}$ (2YQF) & & & \\
\hline & Ankyrin-2 & $\mathrm{O}(4 \mathrm{D} 8 \mathrm{O})$ & & & \\
\hline & Ankyrin-3 & $\mathrm{O}(4 \mathrm{O} 6 \mathrm{X})$ & & & \\
\hline & TNFRSF1A & $\mathrm{O}(1 \mid \mathrm{CH}))$ & & & \\
\hline & TNFRSF16 & & $\mathrm{O}(2 \mathrm{~N} 83)$ & & \\
\hline & TNFRSF25 & O (5YGS) & & & \\
\hline & MALT1 & $\mathrm{O}(2 \mathrm{G} 7 \mathrm{R})$ & $\mathrm{O}(6 \mathrm{GK} 2)$ & & \\
\hline & MyD88 & & & $(3 \mathrm{MOP})^{\mathrm{a}}$ & \\
\hline & NF-kB-1 & $\mathrm{O}(2 \mathrm{DBF})$ & & & \\
\hline & NF-kB-2 & O (2D96) & & & \\
\hline & THOC1 & O (1WXP) & & & \\
\hline & UNC5B & $\mathrm{O}(3 \mathrm{G} 5 \mathrm{~B})$ & O (1WMG) & & \\
\hline \multirow[t]{3}{*}{ DED } & FADD & $\mathrm{O}(2 \mathrm{GF5})$ & & & \\
\hline & Caspase-8 & $\mathrm{O}$ (5JQE) & & $\mathrm{O}(5 \mathrm{~L} 08)$ & $\mathrm{O}(6 \mathrm{AGW})$ \\
\hline & PEA-15 & $\mathrm{O}(6 \mathrm{P} 6 \mathrm{~B})$ & & & \\
\hline \multirow[t]{20}{*}{ CARD } & Caspase-1 & & & O (5FNA) & \\
\hline & Caspase-9 & & O (3YGS) & & \\
\hline & Apaf-1 & O (2YGS) & O (3YGS) & & \\
\hline & ARC & O (4UZO) & & & \\
\hline & ASC & $\mathrm{O}(2 \mathrm{KN} 6)$ & & $\mathrm{O}(6 \mathrm{~N} 1 \mathrm{H})$ & \\
\hline & RAIDD & O (3CRD) & & & \\
\hline & RIPK2 & $\mathrm{O}(2 \mathrm{~N} 7 \mathrm{Z})$ & $\mathrm{O}(2 \mathrm{~N} 83)$ & $\mathrm{O}(6 \mathrm{GGS})$ & \\
\hline & NLRP1 & $\mathrm{O}(4 \mathrm{IFP})$ & & & \\
\hline & NLRC4 & $\mathrm{O}(4 \mathrm{KXF})$ & & $\mathrm{O}(6 \mathrm{~N} 1 \mathrm{I})$ & \\
\hline & NOD1 & $\mathrm{O}(2 \mathrm{~B} 1 \mathrm{~W})$ & $\mathrm{O}(2 \mathrm{NSN})$ & & $\mathrm{O}(2 \mathrm{NSN})$ \\
\hline & NOD4 & O (2MJM) & & & \\
\hline & CARMA1 & O (4LWD) & O (4JUP) & & \\
\hline & ICEBERG & $\mathrm{O}(1 \mathrm{DGN})$ & & & \\
\hline & BinCARD & $\mathrm{O}(4 \mathrm{DWN})$ & & & \\
\hline & CARD8 & $\mathrm{O}(4 \mid \mathrm{KM})$ & & & \\
\hline & CARD9 & $\mathrm{O}(6 \mathrm{E} 26)$ & $\mathrm{O}(6 \mathrm{~N} 2 \mathrm{M})$ & $\mathrm{O}(6 \mathrm{~N} 2 \mathrm{P})$ & $\mathrm{O}(6 \mathrm{~N} 2 \mathrm{M})$ \\
\hline & $\mathrm{BIRC2}$ & O (2L9M) & & & \\
\hline & MAVS & O (2VGQ) & & O (3J6J) & \\
\hline & DDX58 & $\mathrm{O}(2 \mathrm{LWD})$ & & $\mathrm{O}(4 \mathrm{P} 4 \mathrm{H})$ & \\
\hline & Bcl-10 & O (2MB9) & $\mathrm{O}(6 \mathrm{GK} 2)$ & $\mathrm{O}$ (6BZE) & \\
\hline \multirow[t]{13}{*}{ PYD } & ASC & O (2KN6) & & O (3J63) & \\
\hline & NLRP1 & O (1PN5) & & & \\
\hline & NLRP3 & O (3QF2) & & & \\
\hline & NLRP4 & $\mathrm{O}(4 \mathrm{EWI})$ & & & \\
\hline & NLRP6 & $\mathrm{O}(6 \mathrm{NDJ})$ & & $\mathrm{O}(6 \mathrm{NCV})$ & \\
\hline & NLRP7 & O (2KM6) & & & \\
\hline & NLRP10 & $\mathrm{O}(2 \mathrm{M} 5 \mathrm{~V})$ & & & \\
\hline & NLRP12 & $\mathrm{O}(2 \mathrm{~L} 6 \mathrm{~A})$ & O (4XHS) & & \\
\hline & NLRP14 & $\mathrm{O}(4 \mathrm{~N} 1 \mathrm{~L})$ & $\mathrm{O}(4 \mathrm{~N} 1 \mathrm{~J})$ & & $\mathrm{O}(4 \mathrm{~N} 1 \mathrm{~J})$ \\
\hline & POP1 & $\mathrm{O}(2 \mathrm{HM} 2)$ & & & \\
\hline & AlM2 & O (4O7Q) & & $\mathrm{O}(6 \mathrm{MB} 2)$ & \\
\hline & MEFV & $\mathrm{O}(2 \mathrm{MPC})$ & & & \\
\hline & MNDA & O (5WQ6) & & & \\
\hline
\end{tabular}

${ }^{\mathrm{a}}$ Indicates the formation of helical oligomer rather than filaments. commonly interact, the complete DISC complex is thought to form a DISC cluster in lipid rafts (Fig. 3f).

\section{Structure and assembly strategies of the CIDE domain}

Five CIDE domain-containing proteins that perform critical roles in apoptosis and energy metabolism (CIDEA, CIDE-B, CIDE-3 (or CIDE-C and FSP27 in mice), DFF45 (ICAD in mice), and DFF40 (CAD in mice)) were identified and studied ${ }^{9,11,12,14,75-77}$. DFF40 and DFF45, primary executioners for apoptotic DNA fragmentation, contain a CIDE domain in their respective $\mathrm{N}$ terminus ${ }^{12,78}$. The CIDE domain also forms a filamentlike structure that is relevant to its function ${ }^{24}$. In Drosophila, the four CIDE domain-containing proteins DREP1-4 mutually control apoptotic DNA fragmentation $^{13}$. Among these proteins, DREP4 is a homolog of human DFF40, an endonuclease that directly cuts chromosomal DNA during apoptosis ${ }^{79,80}$.

The CIDE domain has an $\alpha / \beta$-roll fold with two $\alpha$ helices and five $\beta$-strands (Fig. 4a) ${ }^{81-83}$. This fold is similar to that of SUMO and ubiquitin, which are small protein modification proteins (Fig. $4 \mathrm{~b})^{84,85}$. The most distinct feature of the CIDE domain is its complementarity between the two opposing acidic and basic surfaces, which can support the formation of homodimeric and heterodimeric filament structures via head-to-tail polymerization (Fig. 4c) ${ }^{86-89}$. The results from a sequence analysis showed that the residues involved in basic and acidic surface formation are conserved in the CIDE domain, indicating that this type of charge distribution, creating two distinctly opposing surfaces, is common in this domain (Fig. 4d).

Representative CIDE domain homodimer formation was revealed via a structural study of FSP27 $\mathrm{CIDE}^{87,90}$. The interface was formed via residues on $\beta 1, \beta 2$, and $\alpha 1$ in one CIDE domain and residues on $\beta 4$ and $\alpha 2$ in another binding CIDE domain (Fig. 4e). As expected, the homodimer interface was composed predominantly of electrostatic interactions formed by R46, R55, and K56 (basic patch) in one FSP27 molecule and E87, D88, and E93 (acidic patch) in the bound FSP27 molecule ${ }^{87,90}$. Although the functional relevance of FSP27 dimerization remains unclear, it may be critical to the biological activity of FSP27. The heterodimerization process of the CIDE domain is similar to that of the homodimerization process, as the CIDE domain has complementarity with the two opposing surfaces. According to the complex structures of the DFF40 CIDE and DFF45 CIDE domains, the basic patch (formed by K9, K18, K32, and R36 in the DFF40 CIDE domain) interacts with the acidic patch (formed by D66, D71, D72, and D74 in the DFF45 CIDE domain), indicating that this oppositely charged surface 
a

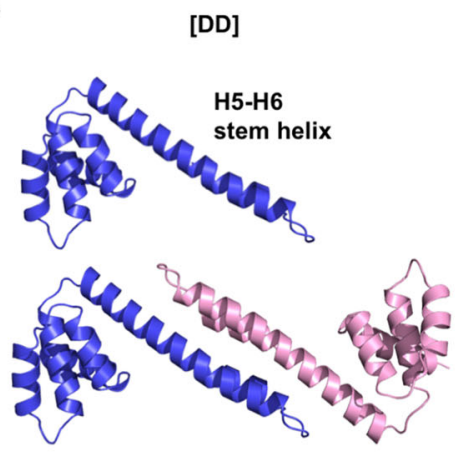

C

[CARD]
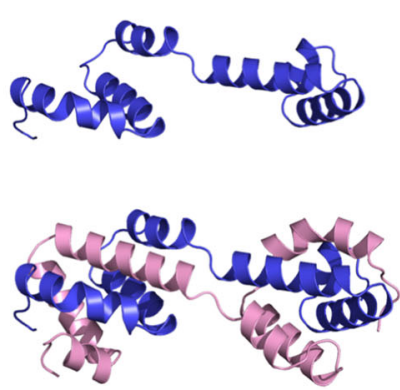

b

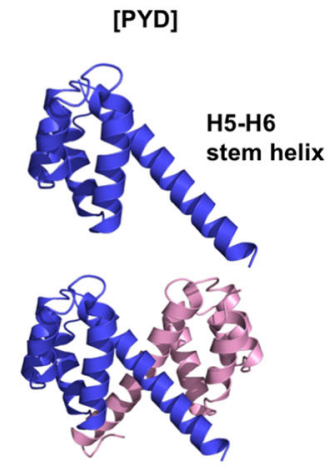

d
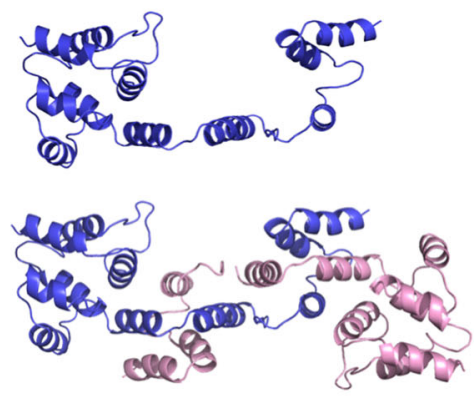

e
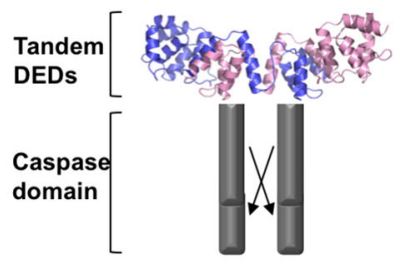

Caspase-8 dimerization

f
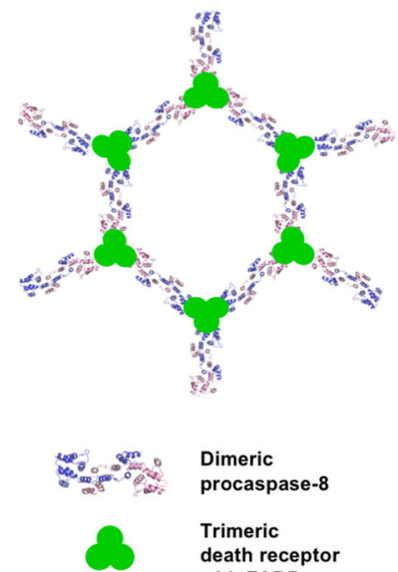

Fig. 3 Domain-swapping-mediated oligomerization in death domain (DD) superfamily members. Representative cases of a DD (Fas DD), b pyrin domain (PYD) (NLRP14), c the caspase recruitment domain (CARD) (COP from frog virus 3), and $\mathbf{d}$ death effector domain (DED) (caspase-8) are shown. Stem helix-mediated dimerization was observed for a DD and $\mathbf{b}$ PYD. The connection of H5 and H6, which serves as a dimerization stem helix, is labeled. A monomer (upper panel) and a stem loop-mediated dimer (lower panel) are shown in a cartoon. Regional domain-swapping-mediated dimerization is shown for CARD (c) and DED (d). A monomer (upper panel) and regional domain-swapping-mediated dimer (lower panel) are shown using a cartoon. e Proposed activation model of caspase-8 by domain-swapping-mediated dimerization of the caspase-8 prodomain (tandem DEDs). f Tentative model of DISC chain assembly via the interaction of the trimeric death receptor bound to the death ligand, adaptor FADD, and domainswapping-mediated dimerized caspase-8.

interaction is a common strategy for CIDE domain dimer formation (Fig. $4 \mathrm{f})^{78}$.

The helical assembly mechanism of the CIDE domain has been recently revealed through structural study of the CIDE domains in DREP4 and DREP2 ${ }^{24,89}$. This newly discovered helix is constructed with ten subunits with a $56.5 \AA$ rise/turn and a diameter of $105 \AA$ (Fig. 4g). This helical oligomer is formed by repetitive head-to-tail oligomerization of highly charged interfaces, detected, and then introduced to homodimeric and heterodimeric CIDE complexes. In the case of DREP4, a negatively charged patch formed by residues D91, E94, D97, E99, and D116 generates massive salt bridges and hydrogen bonds with a positively charged patch comprising residues K51, R59, $\mathrm{K} 60$, and $\mathrm{K} 74$ on the opposite molecule ${ }^{24}$. This helical structure formed by the CIDE domain was also formed in solution, as observed by EM (Fig. 4h). The helical assembly of the CIDE domain was also shown via structural studies of DREP2 and FSP27, indicating that this head-to-tail polymerization via charge-charge interactions may be an assembly mechanism common to CIDE domains ${ }^{24,89}$.

The function of this common head-to-tail helical CIDE domain assembly was studied by observing the apoptotic DNA fragmentation process executed by DFF40 24 . This functional study and previous biochemical studies showed that the CIDE domain-mediated DFF40 dimer has limited nuclease activity, possessing insufficient activity for fast chromosomal DNA fragmentation during apoptosis $^{9,91-93}$. In contrast, the helical filament assembly of DFF40 via its $\mathrm{N}$-terminal CIDE domain can increase the local concentration of DFF40 to impose a compatible distance, functioning as a molecular ruler to efficiently cleave the chromosomal DNA producing apoptotic DNA ladders (Fig. 4i $)^{24}$. The various forms of the CIDE domain as revealed by structural studies are summarized in Table 2 .

\section{Summary and outlook}

The concept of modern signal transduction has been further advanced from classical signal transduction via the 


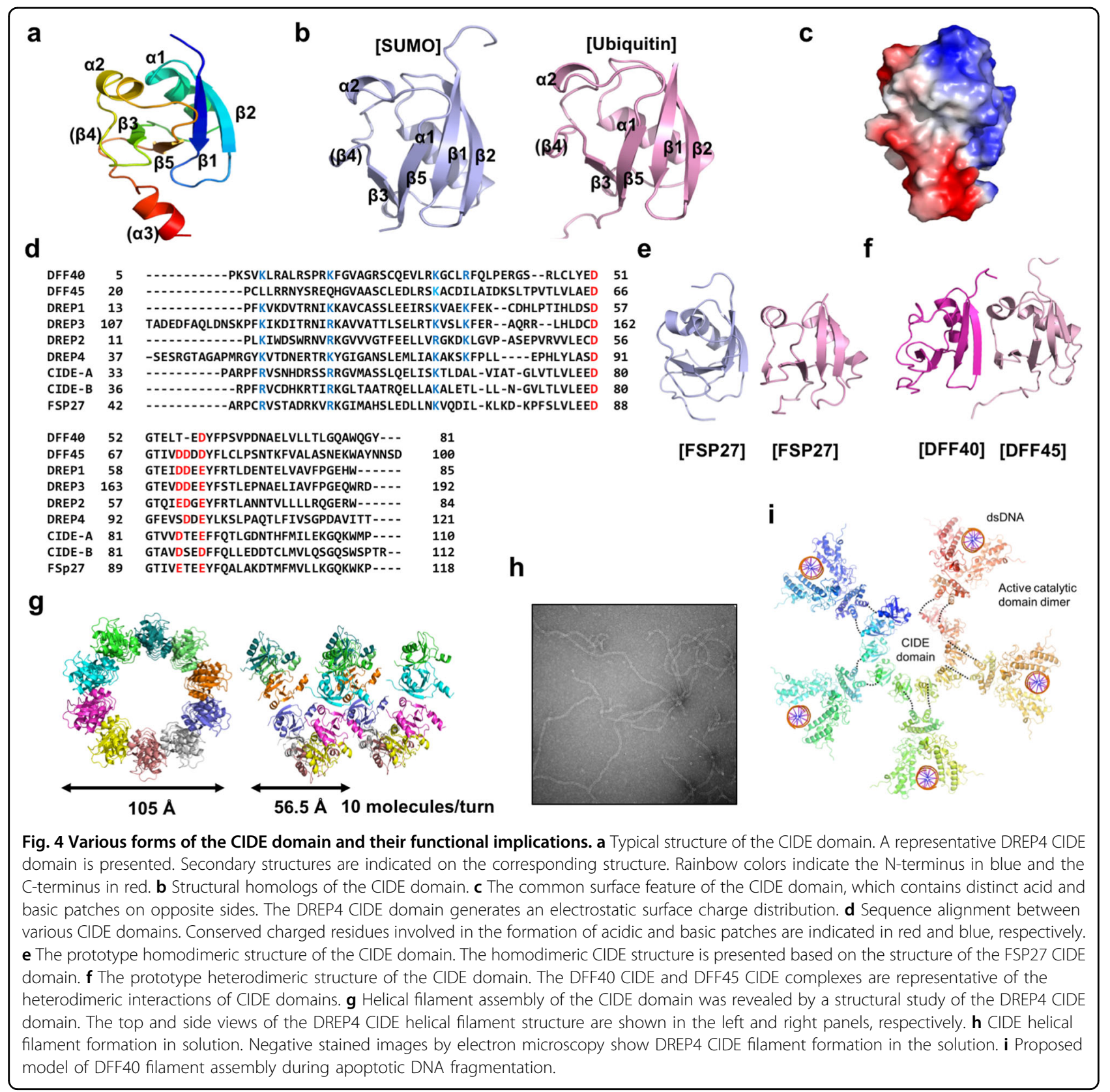

discovery of various SMOCs, which are location-specific, higher-order signaling complexes. This new signaling platform is especially important for cell death and innate immune signaling ${ }^{94}$. In particular, the DD superfamily, composed of the DD, CARD, PYD, and DED subfamilies, mediates SMOC formation using a common helical assembly mechanism. This SMOC can mediate signal transduction, signal amplification, and proximity-induced enzyme activation. DD superfamily-mediated SMOC formation includes caspase activating complexes such as an inflammasome for caspase-1 activation, a PIDDosome for caspase-2 activation, an apoptosome for caspase- 9 activation, and a DISC for caspase- 8 and caspase- 10 activation. A SMOC also includes various signaling platforms, such as a MYDosome for Toll-like signaling and CMD complexes for immune cell signaling. Understanding these huge molecular complexes can explain proximity-mediated allosteric enzyme activation, cooperativity, signal amplification, threshold behavior, and the spatial and temporal control of signal activation and termination.

In addition to helical filament assembly by DD superfamily members, various assembly mechanisms, including several different types of dimerization, have also been observed. Among these formation strategies, domain- 
Table 2 Structures of various forms of CIDE domain.

\begin{tabular}{|c|c|c|c|c|}
\hline CIDE proteins & Monomer & Dimer & Filament & PDB \\
\hline CIDE-A & $\mathrm{O}$ & & & $2 \mathrm{EEL}$ \\
\hline CIDE-B & $\mathrm{O}$ & & & 1D4B \\
\hline FSP27 & & Homodimer & $\mathrm{O}$ & $4 M A C$ \\
\hline DREP2 & & & $\mathrm{O}$ & $4 \mathrm{D} 2 \mathrm{~K}$ \\
\hline DREP4 & & & $\mathrm{O}$ & $5 X P C$ \\
\hline$C A D$ & & Heterodimer & & $1 F 2 R$ \\
\hline ICAD & & Heterodimer & & $1 F 2 R$ \\
\hline DFF40 & & Heterodimer & & $1 \mathrm{IBX}$ \\
\hline DFF45 & & Heterodimer & & $1 \mathrm{IBX}$ \\
\hline
\end{tabular}

swapping-mediated dimerization is a newly identified alternative for DD superfamily dimerization. As DD superfamily-containing proteins are functionally diverse, acting as enzymes or scaffolding adaptors in various cellular signaling pathways, it is unsurprising that these proteins have evolved the capability to form many different types of oligomers using various oligomerization strategies ${ }^{18,24,47,48,95,96}$. Recent studies have demonstrated that CIDE domain-containing proteins also form higherorder SMOCs that perform apoptotic DNA fragmentation functions ${ }^{24}$.

Domain-mediated protein interactions, especially SMOC-mediated signaling transfer in cell death and innate immunity, are important for understanding signal transduction. Signal transduction failure is linked to various human diseases, such as gout, multiple sclerosis, neurodegenerative diseases, diabetes, and Crohn's disease. Understanding the emerging concept of signal transduction by the formation of the large molecular scaffolding center SMOC via DD superfamily members and the CIDE domain may provide a new target for treating human diseases. Blocking SMOC assembly, therefore, may be an interesting avenue for further research.

\section{Acknowledgements}

The authors would like to thank the members of Park laboratory for helping with reference collection and their helpful discussions. This study was supported by the Basic Science Research Program of the National Research Foundation of Korea (NRF) of the Ministry of Education, Science, and Technology (NRF-2017M3A9D8062960 and NRF-2018R1A2B2003635) and a grant from the Korea Healthcare Technology R\&D Project, Ministry of Health \& Welfare, Republic of Korea (HI17C0155).

\section{Conflict of interest}

The authors declare that they have no conflict of interest.

\section{Publisher's note}

Springer Nature remains neutral with regard to jurisdictional claims in published maps and institutional affiliations.
Received: 12 January 2020 Accepted: 31 January 2020.

Published online: 5 March 2020

\section{References}

1. Xia, K., Fu, Z., Hou, L. \& Han, J. D. Impacts of protein-protein interaction domains on organism and network complexity. Genome Res. 18, 1500-1508 (2008).

2. Marcotte, E. M. et al. Detecting protein function and protein-protein interactions from genome sequences. Science $\mathbf{2 8 5}$, 751-753 (1999).

3. Park, H. H. et al. The death domain superfamily in intracellular signaling of apoptosis and inflammation. Ann. Rev. Immunol. 25, 561-586 (2007).

4. Reed, J. C., Doctor, K. S. \& Godzik, A. The domains of apoptosis: a genomics perspective. Sci. STKE 2004, re9 (2004).

5. Palsson-McDermott, E. M. \& O'Neill, L. A. Building an immune system from nine domains. Biochem. Soc. Trans. 35, 1437-1444 (2007).

6. Kwon, D. et al. A comprehensive manually curated protein-protein interaction database for the Death Domain superfamily.Nucleic Acids Res. 40, D331-D336 (2012).

7. Park, H. H. et al. Death domain assembly mechanism revealed by crystal structure of the oligomeric PIDDosome core complex. Cell 128, 533-546 (2007).

8. Kersse, K, Verspurten, J., Vanden Berghe, T. \& Vandenabeele, P. The death-fold superfamily of homotypic interaction motifs. Trends Biochem. Sci. 36, 541-552 (2011).

9. Liu, X., Zou, H., Slaughter, C. \& Wang, X. DFF, a heterodimeric protein that functions downstream of caspase-3 to trigger DNA fragmentation during apoptosis. Cell 89, 175-184 (1997).

10. Sakahira, H., Enari, M. \& Nagata, S. Cleavage of CAD inhibitor in CAD activation and DNA degradation during apoptosis. Nature 391, 96-99 (1998).

11. Park, H. H. Structural insight into CIDE domains: the Janus face of CIDEs, Apoptosis 20, 240-249 (2015).

12. Inohara, N., Koseki, T., Chen, S., Wu, X. \& Nunez, G. CIDE, a novel family of cell death activators with homology to the $45 \mathrm{kDa}$ subunit of the DNA fragmentation factor. Embo J. 17, 2526-2533 (1998).

13. Inohara, N. \& Nunez, G. Genes with homology to DFF/CIDEs found in Drosophila melanogaster. Cell Death Differ. 6, 823-824 (1999).

14. Li, J. Z. et al. Cideb regulates diet-induced obesity, liver steatosis, and insulin sensitivity by controlling lipogenesis and fatty acid oxidation. Diabetes $\mathbf{5 6}$, 2523-2532 (2007).

15. Rubio-Cabezas, O. et al. Partial lipodystrophy and insulin resistant diabetes in a patient with a homozygous nonsense mutation in CIDEC. EMBO Mol. Med. 1, 280-287 (2009).

16. Xu, L., Zhou, L. \& Li, P. CIDE proteins and lipid metabolism. Arterioscler. Thromb. Vasc. Biol. 32, 1094-1098 (2012).

17. Wang, L. et al. The Fas-FADD death domain complex structure reveals the basis of DISC assembly and disease mutations. Nat. Struct. Mol. Biol. 17, 1324-1329 (2010).

18. Fu, T. M. et al. Cryo-EM structure of caspase-8 tandem DED filament reveals assembly and regulation mechanisms of the death-inducing signaling complex. Mol. Cell 64, 236-250 (2016).

19. Li, Y. et al. Cryo-EM structures of ASC and NLRC4 CARD filaments reveal a unified mechanism of nucleation and activation of caspase-1. Proc. Natl Acad. Sci. USA 115, 10845-10852 (2018).

20. Lin, S. C., Lo, Y. C. \& Wu, H. Helical assembly in the MyD88-IRAK4-IRAK2 complex in TLR/L-1R signalling. Nature 465, 885-890 (2010).

21. Lu, A. et al. Molecular basis of caspase-1 polymerization and its inhibition by a new capping mechanism. Nat. Struct. Mol. Biol. 23, 416-425 (2016).

22. Scott, F. L. et al. The Fas-FADD death domain complex structure unravels signalling by receptor clustering. Nature 457, 1019-1022 (2009).

23. Kagan, J. C., Magupalli, V. G. \& Wu, H. SMOCs: supramolecular organizing centres that control innate immunity. Nat. Rev. Immunol. 14, 821-826 (2014).

24. Choi, J. Y. et al. CIDE domains form functionally important higher-order assemblies for DNA fragmentation. Proc. Natl Acad. Sci. USA 114, 7361-7366 (2017).

25. Tartaglia, L. A., Ayres, T. M., Wong, G. H. \& Goeddel, D. V. A novel domain within the $55 \mathrm{kd}$ TNF receptor signals cell death. Cell 74, 845-853 (1993).

26. Beidler, D. R., Tewari, M., Friesen, P. D., Poirier, G. \& Dixit, V. M. The baculovirus p35 protein inhibits Fas- and tumor necrosis factor- induced apoptosis. J. Biol. Chem. 270, 16526-278 (1995). 
27. Brunner, T. et al. Cell-autonomous Fas (CD95)/Fas-ligand interaction mediates activation-induced apoptosis in T-cell hybridomas. Nature 373, 441-444 (1995).

28. Chinnaiyan, A. M., O'Rourke, K., Tewari, M. \& Dixit, V. M. FADD, a novel death domain-containing protein, interacts with the death domain of Fas and initiates apoptosis. Cell 81, 505-512 (1995).

29. Hofmann, K., Bucher, P. \& Tschopp, J. The CARD domain: a new apoptotic signalling motif. Trends Biochem. Sci. 22, 155-156 (1997).

30. Bertin, J. \& DiStefano, P. S. The PYRIN domain: a novel motif found in apoptosis and inflammation proteins. Cell Death Differ. 7, 1273-1274 (2000).

31. Martinon, F., Hofmann, K. \& Tschopp, J. The pyrin domain: a possible member of the death domain-fold family implicated in apoptosis and inflammation. Curr. Biol. 11, R118-R120 (2001).

32. Yin, Q., Fu, T. M., Li, J. \& Wu, H. Structural biology of innate immunity. Annu. Rev. Immunol. 33, 393-416 (2015)

33. Nanson, J. D., Rahaman, M. H., Ve, T. \& Kobe, B. Regulation of signaling by cooperative assembly formation in mammalian innate immunity signalosomes by molecular mimics. Semin. Cell Dev. Biol. 17, 30423-30428 (2018).

34. Chou, J. J., Matsuo, H., Duan, H. \& Wagner, G. Solution structure of the RAIDD CARD and model for CARD/CARD interaction in caspase-2 and caspase-9 recruitment. Cell 94, 171-180 (1998).

35. Hiller, S. et al. NMR structure of the apoptosis- and inflammation-related NALP1 pyrin domain. Structure 11, 1199-1205 (2003).

36. Park, H. H. Structural analyses of death domains and their interactions. Apoptosis 16, 209-220 (2011).

37. Park, H. H. PYRIN domains and their interactions in the apoptosis and inflammation signaling pathway. Apoptosis 17, 1247-1257 (2012).

38. Park, H. H. Caspase recruitment domains for protein interactions in cellular signaling (Review). Int. J. Mol. Med. 43, 1119-1127 (2019).

39. Qin, $H$. et al. Structural basis of procaspase-9 recruitment by the apoptotic protease-activating factor 1. Nature 399, 549-557 (1999).

40. Jang, T. H. et al. Crystal structure of caspase recruiting domain (CARD) of apoptosis repressor with CARD (ARC) and its implication in inhibition of apoptosis. Sci. Rep. 5, 9847 (2015).

41. Xiao, T., Towb, P., Wasserman, S. A. \& Sprang, S. R. Three-dimensional structure of a complex between the death domains of Pelle and Tube. Cell 99, 545-555 (1999).

42. Jang, T. H. et al. Structural study of the RIPoptosome core reveals a helical assembly for kinase recruitment. Biochemistry 53, 5424-5431 (2014).

43. Lin, Z. et al. Structural basis of death domain signaling in the p75 neurotrophin receptor. Elife 4, e11692 (2015).

44. Jang, T. H., Park, J. H. \& Park, H. H. Novel disulfide bond-mediated dimerization of the CARD domain was revealed by the crystal structure of CARMA1 CARD. PLOS ONE 8, e79778 (2013)

45. Handa, N. et al. Structure of the UNC5H2 death domain. Acta Crystallogr. D Biol. Crystallogr. 62, 1502-1509 (2006)

46. Schlauderer, F. et al. Molecular architecture and regulation of BCL10-MALT1 filaments. Nat. Commun. 9, 4041 (2018).

47. Peisley, A., Wu, B., Xu, H., Chen, Z. J. \& Hur, S. Structural basis for ubiquitinmediated antiviral signal activation by RIG-I. Nature 509, 110-114 (2014).

48. Lu, A. et al. Unified polymerization mechanism for the assembly of ASCdependent inflammasomes. Cell 156, 1193-1206 (2014).

49. Shen, C. et al. Molecular mechanism for NLRP6 inflammasome assembly and activation. Proc. Natl Acad. Sci. USA 116, 2052-2057 (2019).

50. Pellegrini, E. et al. RIP2 filament formation is required for NOD2 dependent NFkappaB signalling. Nat. Commun. 9, 4043 (2018).

51. Bae, J. Y. \& Park, H. H. Crystal structure of NALP3 protein pyrin domain (PYD) and its implications in inflammasome assembly. J. Biol. Chem. 286, 39528-39536 (2011).

52. Davis, B. K., Wen, H. \& Ting, J. P. The inflammasome NLRs in immunity, inflammation, and associated diseases. Annu. Rev. Immunol. 29, 707-735 (2011).

53. Dostert, $C$. et al. Innate immune activation through Nalp3 inflammasome sensing of asbestos and silica. Science 320, 674-677 (2008).

54. Franchi, L., Eigenbrod, T., Munoz-Planillo, R. \& Nunez, G. The inflammasome: a caspase-1-activation platform that regulates immune responses and disease pathogenesis. Nat. Immunol. 10, 241-247 (2009).

55. Schroder, K. \& Tschopp, J. The inflammasomes. Cell 140, $821-832$ (2010).

56. Sborgi, L. et al. Structure and assembly of the mouse ASC inflammasome by combined NMR spectroscopy and cryo-electron microscopy. Proc. Natl Acad. Sci. USA 112, 13237-13242 (2015).
57. Shen, C., Sharif, H., Xia, S. \& Wu, H. Structural and mechanistic elucidation of inflammasome signaling by cryo-EM. Curr. Opin. Struct. Biol. 58, 18-25 (2019).

58. Zhang, L. et al. Cryo-EM structure of the activated NAIP2-NLRC4 inflammasome reveals nucleated polymerization. Science 350, 404-409 (2015).

59. Yang, S., Levine, H., Onuchic, J. N. \& Cox, D. L. Structure of infectious prions: stabilization by domain swapping. FASEB J. 19, 1778-1782 (2005).

60. Liu, C., Sawaya, M. R. \& Eisenberg, D. beta(2)-microglobulin forms threedimensional domain-swapped amyloid fibrils with disulfide linkages. Nat. Struct. Mol. Biol. 18, 49-55 (2011).

61. Janowski, R., Abrahamson, M., Grubb, A. \& Jaskolski, M. Domain swapping in Ntruncated human cystatin C. J. Mol. Biol. 341, 151-160 (2004).

62. Liu, Y., Hart, P. J., Schlunegger, M. P. \& Eisenberg, D. The crystal structure of a 3D domain-swapped dimer of RNase A at a 2.1-A resolution. Proc. Natl Acad. Sci. USA 95, 3437-3442 (1998).

63. Hirota, S. et al. Cytochrome c polymerization by successive domain swapping at the C-terminal helix. Proc. Natl Acad. Sci. USA 107, 12854-12859 (2010).

64. Fagagnini, A. et al. Onconase dimerization through 3D domain swapping: structural investigations and increase in the apoptotic effect in cancer cells. Biochem. J. 474, 3767-3781 (2017)

65. Eibl, C., Hessenberger, M., Wenger, J. \& Brandstetter, H. Structures of the NLRP14 pyrin domain reveal a conformational switch mechanism regulating its molecular interactions. Acta Crystallogr. D Biol. Crystallogr. 70, 2007-2018 (2014).

66. Park, H. H. Molecular basis of dimerization of initiator caspase was revealed by crystal structure of caspase-8 pro-domain. Cell Death Differ. 26, 1213-1220 (2019).

67. Shen, $C$. et al. Structural basis for dimerization of the death effector domain of the F122A mutant of Caspase-8. Sci. Rep. 8, 16723 (2018).

68. Kim, C. M. et al. Structural transformation-mediated dimerization of caspase recruitment domain revealed by the crystal structure of CARD-only protein in frog virus 3. J. Struct. Biol. 205, 189-195 (2019).

69. Coussens, N. P., Mowers, J. C., McDonald, C., Nunez, G. \& Ramaswamy, S. Crystal structure of the Nod1 caspase activation and recruitment domain. Biochem. Biophys. Res. Commun. 353, 1-5 (2007).

70. Bennett, M. J., Choe, S. \& Eisenberg, D. Domain swapping: entangling alliances between proteins. Proc. Natl Acad. Sci. USA 91, 3127-3131 (1994).

71. Liu, Y. \& Eisenberg, D. 3D domain swapping: as domains continue to swap. Protein Sci. 11, 1285-1299 (2002).

72. Dickens, L. S. et al. A death effector domain chain DISC model reveals a crucial role for caspase-8 chain assembly in mediating apoptotic cell death. Mol. Cell 47, 291-305 (2012).

73. Majkut, J. et al. Differential affinity of FLIP and procaspase 8 for FADD's DED binding surfaces regulates DISC assembly. Nat. Commun. 5, 3350 (2014).

74. Singh, N., Hassan, A. \& Bose, K. Molecular basis of death effector domain chain assembly and its role in caspase-8 activation. FASEB J. 30, 186-200 (2016).

75. Lin, S. C. \& Li, P. CIDE-A, a novel link between brown adipose tissue and obesity. Trends Mol. Med. 10, 434-439 (2004).

76. Zhou, Z. et al. Cidea-deficient mice have lean phenotype and are resistant to obesity. Nat. Genet. 35, 49-56 (2003).

77. Enari, M. et al. A caspase-activated DNase that degrades DNA during apoptosis, and its inhibitor ICAD. Nature 391, 43-50 (1998).

78. Zhou, P., Lugovskoy, A. A., McCarty, J. S., Li, P. \& Wagner, G. Solution structure of DFF40 and DFF45 N-terminal domain complex and mutual chaperone activity of DFF40 and DFF45. Proc. Natl Acad. Sci. USA 98, 6051-6055 (2001).

79. Mukae, N. et al. Identification and developmental expression of inhibitor of caspase-activated DNase (ICAD) in Drosophila melanogaster. J. Biol. Chem. 275, 21402-21408 (2000).

80. Yokoyama, H. et al. A novel activation mechanism of caspase-activated DNase from Drosophila melanogaster. J. Biol. Chem. 275, 12978-12986 (2000).

81. Otomo, T., Sakahira, H., Uegaki, K., Nagata, S. \& Yamazaki, T. Structure of the heterodimeric complex between CAD domains of CAD and ICAD. Nat. Struct. Biol. 7, 658-662 (2000).

82. Uegaki, K. et al. Structure of the CAD domain of caspase-activated DNase and interaction with the CAD domain of its inhibitor. J. Mol. Biol. 297, 1121-1128 (2000).

83. Fukushima, K. et al. Solution structure of the DFF-C domain of DFF45/ICAD. A structural basis for the regulation of apoptotic DNA fragmentation. J. Mol. Biol. 321, 317-327 (2002).

84. Huang, W. C., Ko, T. P., Li, S. S. \& Wang, A. H. Crystal structures of the human SUMO-2 protein at $1.6 \mathrm{~A}$ and $1.2 \mathrm{~A}$ resolution: implication on the functional differences of SUMO proteins. Eur. J. Biochem. 271, 4114-4122 (2004). 
85. Swanson, K. A., Hicke, L. \& Radhakrishnan, I. Structural basis for monoubiquitin recognition by the Ede1 UBA domain. J. Mol. Biol. 358, 713-724 (2006).

86. Lee, S. M. \& Park, H. H. In vitro analysis of the complete CIDE domain interactions of the Drep system in fly. Apoptosis 19, 428-435 (2014).

87. Lee, S. M., Jang, T. H. \& Park, H. H. Molecular basis for homo-dimerization of the CIDE domain revealed by the crystal structure of the CIDE-N domain of FSP27. Biochem. Biophys. Res. Commun. 439, 564-569 (2013).

88. Lee, S. M. \& Park, H. H. General interaction mode of CIDE:CIDE complex revealed by a mutation study of the Drep2 CIDE domain. FEBS Lett. 587, 854-859 (2013).

89. Ha, H. J. \& Park, H. H. Crystal structure and mutation analysis revealed that DREP2 CIDE forms a filament-like structure with features differing from those of DREP4 CIDE. Sci. Rep. 8, 17810 (2018).

90. Sun, Z. et al. Perilipin1 promotes unilocular lipid droplet formation through the activation of Fsp27 in adipocytes. Nat. Commun. 4, 1594 (2013).
91. Liu, X., Zou, H., Widlak, P., Garrard, W. \& Wang, X. Activation of the apoptotic endonuclease DFF40 (caspase-activated DNase or nuclease) Oligomerization and direct interaction with histone H1. J. Biol. Chem. 274, 13836-13840 (1999).

92. Nagata, S. Apoptotic DNA fragmentation. Exp. Cell Res. 256, 12-18 (2000).

93. Inohara, N., Koseki, T., Chen, S., Benedict, M. A. \& Nunez, G. Identification of regulatory and catalytic domains in the apoptosis nuclease DFF40/CAD. J. Biol. Chem. 274, 270-274 (1999).

94. $\mathrm{Wu}, \mathrm{H}$. Higher-order assemblies in a new paradigm of signal transduction. Cell 153, 287-292 (2013).

95. Chun, H. J. et al. Pleiotropic defects in lymphocyte activation caused by caspase-8 mutations lead to human immunodeficiency. Nature 419, 395-399 (2002).

96. Wang, L., Du, F. \& Wang, X. TNF-alpha induces two distinct caspase-8 activation pathways. Cell 133, 693-703 (2008). 